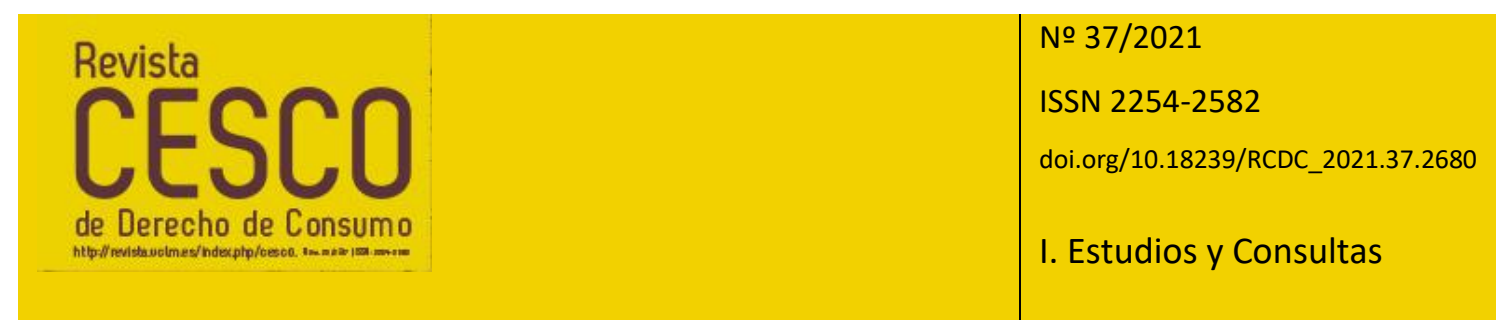

\title{
EL CONCEPTO DE CONSUMIDOR VULNERABLE EN EL TEXTO REFUNDIDO DE LA LEY GENERAL PARA LA DEFENSA DE LOS CONSUMIDORES Y USUARIOS*
}

\author{
Manuel Jesús Marín López \\ Catedrático de Derecho Civil \\ Centro de Estudios de Consumo \\ Universidad de Castilla-La Mancha
}

Title: The concept of vulnerable consumer in the Consolidated Text of the General Law for the Defense of Consumers and Users

Resumen: El RD-ley 1/2021 introduce el concepto de "consumidor vulnerable" en el art. 3.2 TRLGDCU. En este trabajo se explica la definición de "consumidor vulnerable", relacionándolo con el concepto de "consumidor" del art. 3.1 TRLGDCU, y se exponen las distintas concepciones del consumidor vulnerable que en realidad se recogen en el TRLGDCU.

Palabras clave: Concepto de consumidor vulnerable, concepciones consumidor, Ley de consumidores, TRLGDCU.

Abstract: The RD-law 1/2021 introduces the concept of "vulnerable consumer" in art. 3.2 TRLGDCU. This paper explains the definition of "vulnerable consumer", relating it to the concept of "consumer" in art. 3.1 TRLGDCU, and the different

\footnotetext{
* Trabajo realizado en el marco del Proyecto de Investigación PGC2018-098683-B-I00, del Ministerio de Ciencia, Innovación y Universidades (MCIU) y la Agencia Estatal de Investigación (AEI) cofinanciado por el Fondo Europeo de Desarrollo Regional (FEDER) titulado "Protección de consumidores y riesgo de exclusión social", dirigido por Ángel Carrasco Perera y Encarna Cordero Lobato; a la Ayuda para la financiación de actividades de investigación dirigidas a grupos de la UCLM Ref.: 2020-GRIN-29156, denominado "Grupo de Investigación del Profesor Ángel Carrasco" (GIPAC) y a la ayuda para la realización de proyectos de investigación científica y transferencia de tecnología, de la Junta de Comunidades de Castilla-La Mancha cofinanciadas por el Fondo Europeo de Desarrollo Regional (FEDER) para el Proyecto titulado "Protección de consumidores y riesgo de exclusión social en Castilla-La Mancha" (PCRECLM) con Ref.: SBPLY/19/180501/000333 dirigido por Ángel Carrasco Perera y Ana Isabel Mendoza Losana.
} 
conceptions of the vulnerable consumer that are actually included in the TRLGDCU are exposed.

Key words: Concept of vulnerable consumer, consumer conceptions, Consumer Law, TRLGDCU.

Sumario: 1. Introducción. 2. No cambia el concepto de "consumidor" en el artículo 3.1. TRLGDCU. 3. El concepto de "persona consumidora vulnerable" del artículo 3.2. TRLGDCU. 4. ¿El consumidor vulnerable es un subtipo de consumidor del artículo 3.1. TRLGDCU o puede ser consumidor vulnerable una persona que no es consumidora en el sentido del artículo 3.1.? 5. El TRLGDCU tiene varios conceptos de "consumidor vulnerable". 6. El consumidor vulnerable como contratante. 7. El consumidor vulnerable como destinatario de prácticas comerciales, publicidad e información precontractual. 8. Bibliografía.

\section{Introducción}

El Real Decreto-ley 1/2021, de 19 de enero, de protección de los consumidores y usuarios frente a situaciones de vulnerabilidad social y económica, introduce numerosos cambios en el Texto Refundido de la Ley General para la Defensa de los Consumidores y Usuarios (TRLGDCU). El más importante, sin duda, es la modificación del artículo 3, que regulaba el concepto de consumidor y que ahora contiene también, en su apartado segundo, una definición de persona consumidora vulnerable.

En este trabajo se analiza el nuevo concepto de consumidor vulnerable del art. 3.2 TRLGDCU. Aunque para ello será necesario aludir también a otros preceptos del TRLGDCU.

\section{No cambia el concepto de "consumidor" en el artículo 3.1. TRLGDCU}

El RD-ley 1/2021 da una nueva redacción al art. 3 TRLGDCU. Antes este precepto, cuya rúbrica era "concepto general de consumidor y de usuario", tenía dos párrafos. Tras la reforma cambia la rúbrica ("conceptos de consumidor y usuario y de persona consumidora vulnerable") y se divide en dos apartados: el primer define al consumidor y usuario y el segundo a la persona consumidora vulnerable.

Es importante destacar que el RD-ley no modifica el concepto de consumidor y usuario. Los dos párrafos del nuevo art. 3.1 TRLGCU mantienen la misma redacción que el viejo art. 3. Existe un único cambio, que carece de trascendencia jurídica (en el párrafo primero, donde antes se decía "a efectos de esta norma" ahora se dice "a efectos de esta ley").

En consecuencia, el concepto "general" de consumidor no cambia, y debe ser interpretado en los mismos términos en que se hacía antes de la reforma (sobre el particular, v. mi trabajo "El «nuevo» concepto de consumidor y empresario tras la 
Ley 3/2014, de reforma del TRLGDCU", Revista CESCO de Derecho de Consumo, 2014, no 9, pp. 9 y ss.).

\section{El concepto de "persona consumidora vulnerable" del artículo 3.2. TRLGDCU}

El nuevo art. 3.2 TRLGDCU establece lo siguiente:

"Asimismo, a los efectos de esta ley y sin perjuicio de la normativa sectorial que en cada caso resulte de aplicación, tienen la consideración de personas consumidoras vulnerables respecto de relaciones concretas de consumo, aquellas personas físicas que, de forma individual o colectiva, por sus características, necesidades o circunstancias personales, económicas, educativas o sociales, se encuentran, aunque sea territorial, sectorial o temporalmente, en una especial situación de subordinación, indefensión o desprotección que les impide el ejercicio de sus derechos como personas consumidoras en condiciones de igualdad".

El art. 3.2 TRLGDCU establece el concepto "general" de consumidor vulnerable. Pues expresamente admite que pueden existir otras definiciones de consumidor vulnerable en la "normativa sectorial" (así, por ejemplo, los arts. 9 del RD-ley 8/2020 y 11 del RD-ley $11 / 2020$ definen cuándo una persona está en situación de vulnerabilidad económica a los efectos de concederle el derecho a la moratoria hipotecaria).

Llama la atención también el término utilizado: "persona consumidora vulnerable". La expresión acogida no parece adecuada. No tiene sentido que el art. 3.1 aluda al "consumidor" y el art. 3.2 a la "persona consumidora vulnerable". Un uso lógico y coherente de los términos debería implicar que si en el art. 3.1 se establece el concepto de "consumidor", el art. 3.2 acoja el de "consumidor vulnerable". La otra opción sería que en ambos apartados se usara la fórmula "persona consumidora" y "persona consumidora vulnerable" (como hace la Ley vasca 6/2003, de Estatuto de las Personas Consumidoras y Usuarias). No creo que esta sea una buena decisión. Por ello, creo que hubiera sido mejor que el art. 3.2 se refiera, sin más, al "consumidor vulnerable" en lugar de a la "persona consumidora vulnerable".

El consumidor vulnerable es una persona física. No tienen esta consideración, por tanto, ni las personas jurídicas, ni los entes sin personalidad, que sin embargo sí pueden reputarse consumidores a los efectos del art. 3.1 TRLGDCU. El consumidor vulnerable es una persona física, aunque la especial situación de subordinación, indefensión o desprotección puede producirse por su pertenencia a un colectivo.

Lo que caracteriza al consumidor vulnerable es que es una persona que está en "una especial situación de subordinación, indefensión o desprotección que les impide el ejercicio de sus derechos como personas consumidoras en condiciones de igualdad". Por la situación en que se encuentra esa persona, no puede ejercitar sus derechos en condiciones de igualdad. No es necesario, por tanto, que esté impedida de ejercitar 
sus derechos. Quizás sí pueda ejercitarlos, pero no en condiciones de igualdad, esto es, en las mismas condiciones que los podría ejercitar si no estuviera en esa situación de subordinación, indefensión o desprotección. Por lo tanto, el consumidor vulnerable se define por comparación con el consumidor "normal", que es el que no está en esa situación especial de vulnerabilidad. A diferente de este, aquel está en una situación de desigualdad en el ejercicio de los derechos, lo que significa que para él es más costoso, difícil o complicado ese ejercicio o que incluso ese ejercicio resulta imposible.

La "especial situación de subordinación, indefensión o desprotección" en que se encuentra el consumidor vulnerable puede obedecer a múltiples causas. El art. 3.2 TRLGDCU hace una enumeración amplísima: la persona está en esa situación "por sus características, necesidades o circunstancias personales, económicas, educativas o sociales". Cualquier circunstancia imaginable tiene cabida en este elenco. Además, puede encontrarse en esa situación "territorial, sectorial o temporalmente", lo que viene a extender todavía más la definición de consumidor vulnerable. Pues un sujeto puede ser vulnerable únicamente cuando vive en un territorio ( $y$ no en otro), cuando contrata en un determinado sector (pero no en otro), o solamente en un determinado momento ( $y$ no en otros).

Estas características que hacen que una persona esté en situación de desprotección pueden concurrir "de forma individual o colectiva". Hay determinados colectivos que, por su propia naturaleza, convierten a sus miembros en consumidores vulnerables. La Exposición de Motivos del RD-ley 1/2021 enumera algunos de estos colectivos: personas de determinada edad, sexo, origen nacional o étnico, lugar de procedencia, las personas alérgicas o con algún tipo de intolerancia alimenticia, las víctimas de violencia de género, las familias monoparentales, las personas desempleadas, las personas con algún tipo de discapacidad, las personas enfermas, las minorías étnicas o lingüísticas, las personas desplazadas temporalmente de su residencia habitual, la población migrante o los solicitante de protección internacional. Pero estos sujetos no son consumidores vulnerables siempre y en todo caso, sino únicamente cuando en una concreta relación de consumo no pueden ejercitar sus derechos en condiciones de igualdad en comparación con otro sujeto que no pertenezca a ese colectivo.

En consecuencia, el consumidor vulnerable es un concepto dinámico. La Exposición de Motivos lo explica con claridad: "una persona puede ser considerada vulnerable en un determinado ámbito de consumo, pero no en otros. Además, esa condición de vulnerabilidad podrá variar a lo largo del tiempo según puedan hacerlo las condiciones que la determinan, tanto las de tipo personal como las sociales o de contexto". Por eso, cualquier persona puede ser vulnerable en algún momento de su vida.

Otro rasgo que caracteriza a la definición de consumidor vulnerable es que una persona física puede calificarse como tal "respecto de relaciones concretas de consumo" (art. 3.2 TRLGDCU). Esto significa dos cosas. En primer lugar, que en una 
determinada relación de consumo un sujeto puede ser un consumidor vulnerable, pero no en otra relación de consumo, en la que no actúa en esa situación de desprotección o indefensión. En segundo lugar, que el art. 3.2 define al consumidor vulnerable en las relaciones de consumo. La relación de consumo se define por su origen contractual: hay un sujeto que adquiere bienes o servicios de un empresario. Por lo tanto, cuando ese sujeto que adquiere bienes o servicios está en una especial situación de subordinación, indefensión o desprotección, se reputará "consumidor vulnerable", como prevé en art. 3.2 TRLGDCU.

\section{4. ¿EI consumidor vulnerable es un subtipo de consumidor del artículo 3.1 . TRLGDCU o puede ser consumidor vulnerable una persona que no es consumidora en el sentido del artículo 3.1.?}

Cabe preguntarse si el consumidor vulnerable al que se refiere el art. 3.2 TRLGDCU es una clase de consumidor del art. 3.1, o por el contrario el puede calificarse como consumidor vulnerable una persona que no tiene cabida en el concepto de consumidor del art. 3.1? La ordenación del art. 3 TRLGDCU no sirve para aclarar esta cuestión, pues el precepto se limita a exponer un concepto de consumidor y de consumidor vulnerable en sus dos apartados, sin establecer ningún tipo de relación entre ambos.

El art. 3.1 TRLGDCU define al consumidor como al que celebra un contrato con un propósito ajeno a una actividad empresarial o profesional. Conforme a una primera interpretación, el consumidor vulnerable es una clase de consumidor del art. 3.1. Por ello, todo consumidor vulnerable es, por definición, un consumidor en el sentido del art. 3.1 TRLGDCU. Esta tesis puede apoyarse en la expresión "respecto de relaciones concretas de consumo" incluida en el art. 3.2 TRLGDCU, que viene a aclarar que, para calificar a un sujeto como consumidor vulnerable, ha de estarse a la concreta relación (contractual) de consumo. Es preciso, pues, que al adquirir un bien o servicio esa persona actúe con un propósito ajeno a una actividad empresarial o profesional, y además se encuentre en una situación de desprotección que le impida ejercitar sus derechos como consumidor en condiciones de igualdad. Es vulnerable porque no puede ejercitar sus derechos "como consumidor", de donde resulta que ha de ser "consumidor" en el sentido del art. 3.1. Esta interpretación puede tener su apoyo en la Exposición de Motivos del RD-ley 1/2021, cuando señala que se trata de garantizar "con un grado mayor de protección a los derechos en determinados supuestos en los que la persona consumidora se ve afectada por una especial situación de vulnerabilidad". El consumidor vulnerable es un consumidor, pero algo más que un consumidor (un consumidor especial); por eso ha de tener un grado mayor de protección.

Pero cabe defender una segunda interpretación: el consumidor vulnerable del art. 3.2 TRLGDCU no tiene por qué ser un consumidor en el sentido del art. 3.1 TRLGDCU. Así, un consumidor vulnerable es el que se encuentra en la situación de inferioridad o desprotección que describe el art. 3.2, aunque no celebre un contrato un 
empresario al margen de su actividad empresarial o profesional. El argumento decisivo en favor de esta tesis es que el art. 3.2, ni establece expresamente que el consumidor vulnerable sea una clase de consumidor (un subtipo), ni exige que el consumidor vulnerable actué con un propósito ajeno a una actividad empresarial o profesional.

A mi juicio, el consumidor vulnerable del art. 3.2 TRLGDCU es un subtipo de consumidor. De manera que solo es consumidor vulnerable en el sentido del art. 3.2 el consumidor que, actuando al margen de su actividad empresarial o profesional, está en una especial situación de subordinación, indefensión o desprotección que le impide el ejercicio de sus derechos como persona consumidora en condiciones de igualdad.

Pero la realidad es que la asunción de una u otra tesis no tiene consecuencias prácticas, porque al margen del concepto de consumidor vulnerable que se contiene en el art. 3.2 TRLGDCU, este Texto Refundido se refiere al consumidor vulnerable en otros preceptos con un significado diferente.

\section{EI TRLGDCU tiene varios conceptos de "consumidor vulnerable"}

Es cierto que el TRLGDCU contiene una única definición de consumidor vulnerable, recogida en el art. 3.2. Pero en otros preceptos se alude al consumidor vulnerable en otro sentido, con un significado diferente.

Repárese que exactamente lo mismo sucede con el concepto de consumidor. El art. 3.1 define al consumidor por su origen contractual: es el sujeto que contrata con un empresario con un propósito ajeno a una actividad empresarial o profesional. Por tanto, el consumidor "en sentido estricto" es siempre un sujeto que celebra un contrato. Pero para el TRLGDCU también es consumidor la persona que tiene derecho a que se proteja su salud y seguridad, que tiene derecho a ser informado y a recibir una educación adecuada, o que tiene derecho a ser reparado de los daños sufridos mediante la correspondiente indemnización. Estos son "derechos básicos de los consumidores", según el art. 8.1 TRLGDCU. Aquí se alude al consumidor no como contratante, sino como ciudadano. A esta concepción "amplia" de consumidor es la que se asume en la frase "todos somos consumidores".

Existe también otro concepto de consumidor, en relación con las prácticas comerciales desleales: el consumidor medio. La ley prohíbe los actos de competencia desleal. Es desleal la práctica comercial que distorsiona o puede distorsionar de manera significativa el comportamiento económico del consumidor medio (art. 4.1 LCD). Desde hace años el TJUE ha definido al consumidor medio como a un consumidor normalmente informado y razonablemente atento y perspicaz. Repárese que aquí el consumidor no es un sujeto que contrata, sino un simple destinatario de información o publicidad. Tampoco puede ser cualquier persona (no coincide con el concepto "amplio" de consumidor). Es únicamente un grupo concreto de destinatarios 
de información o publicidad, en los que concurren ciertas cualidades (están normalmente informados y son razonablemente atentos y perspicaces).

Lo que acaba de decirse respecto al concepto de consumidor es predicable también del concepto de consumidor vulnerable.

Hay al menos tres conceptos legales de consumidor vulnerable. En primer lugar, está el concepto "estricto" del art. 3.2 TRLGDCU. Como ya he señalado, es el consumidor que contrata al margen de su actividad empresarial o profesional y además lo hace en una situación de inferioridad o desprotección que le impide el ejercicio de los derechos como consumidor en condiciones de igualdad.

Pero existe un segundo concepto de consumidor vulnerable (concepto "amplio"), recogido en la nueva redacción del art. 8 dada por el RD-ley 1/2021. Esta norma enumera los derechos básicos de los consumidores y de los consumidores vulnerables. Tanto unos como otros tienen derecho a la información y educación, a la protección de su salud y seguridad, a la indemnización de los daños sufridos, etc. En este caso, son consumidores vulnerables las personas en quienes concurre alguna de las circunstancias enunciadas en el art. 3.2 TRLGDCU (personas físicas que por sus características, necesidades o circunstancias personales, económicas, educativas o sociales, se encuentran, aunque sea territorial, sectorial o temporalmente, en una especial situación de subordinación, indefensión o desprotección que les impide el ejercicio de sus derechos como personas consumidoras en condiciones de igualdad), aunque no hayan celebrado un contrato con un empresario. Si el consumidor goza de los derechos reconocidos en este art. 8 TRLGDCU, también los disfruta el consumidor vulnerable, con el añadido de que estos derechos del consumidor vulnerable "gozarán de mayor atención" (art. 8.2), lo que significa que serán especialmente recogidos en la normativa reglamentaria y sectorial, y que los poderes públicos "promocionarán políticas y actuaciones tendentes a garantizar sus derechos en condiciones de igualdad... tratando de evitar, en cualquier caso, trámites que puedan dificultar el ejercicio de los mismos" (art. 8.2).

También existe el consumidor especialmente vulnerable a una práctica comercial. Las prácticas comerciales que, dirigidas a los consumidores en general, únicamente sean susceptibles de distorsionar de forma significativa, en un sentido que el empresario o profesional pueda prever razonablemente, el comportamiento económico de un grupo claramente identificable de consumidores o usuarios especialmente vulnerables a tales prácticas o al bien o servicio al que se refieran, por presentar una discapacidad, por tener afectada su capacidad de comprensión o por su edad o su credulidad, se evaluarán desde la perspectiva del miembro medio de ese grupo (art. 4.3 LCD). El parámetro para medir la deslealtad de la práctica comercial es el de ese consumidor vulnerable, y no el de un consumidor normal. 
En definitiva, cabe aproximarse al consumidor vulnerable desde una doble perspectiva: como contratante y como sujeto destinatario de prácticas comerciales, publicidad e información precontractual.

\section{El consumidor vulnerable como contratante}

Ya se ha indicado que el art. 3.2 TRLGDCU define al consumidor vulnerable como al consumidor que, al celebrar un contrato con un empresario al margen de una actividad empresarial, lo hace en una situación de indefensión o desprotección que le impide ejercitar sus derechos como consumidor en condiciones de igualdad.

Hay que advertir en la actual normativa contractual de consumo no hay lugar para este "consumidor vulnerable". Así, por ejemplo, en la normativa reguladora de las garantías en la venta de bienes de consumo (arts. 114 y ss. TRLGDCU) todos los consumidores son iguales, sin que se trate de manera especial al "consumidor vulnerable". Lo mismo sucede en las normas que regulan el crédito al consumo, el crédito inmobiliario, las ventas a distancia o fuera de establecimiento, los viajes combinados o el aprovechamiento por tuno de bienes inmuebles. Así ocurre también en materia de cláusulas abusivas, en lo que al control de incorporación y control de contenido se refiere. En relación con el control de contenido, es abusiva la cláusula que, en contra de las exigencias de buena fe, causa un desequilibrio importante en los derechos y obligaciones de las partes. La abusividad se mide en términos objetivos: que exista un desequilibrio en derecho contrario a la buena fe. La "voluntad virtual del consumidor" a la que aluden algunas sentencias del TJUE no es un nuevo parámetro subjetivo para juzgar la abusividad, por lo que a esos efectos es indiferente si el consumidor adherente está en situación de vulnerabilidad (por ejemplo, es una persona anciana o un analfabeto funcional).

Hasta la fecha la normativa sectorial de consumo no concede al contratante que sea consumidor vulnerable más derechos por esa circunstancia. Pero para contratos ya celebrados y en vigor sí se han dictado normas específicas para proteger a determinados sujetos en situaciones de vulnerabilidad económica: la normativa COVID (desde el RD-ley 8/2020 en adelante) le permite suspender el pago del arrendamiento, de los préstamos hipotecarios o al consumo o de los servicios básicos esenciales, como la luz). Ahora bien, no podemos ignorar que esta normativa COVID ha utilizado un concepto de contratante vulnerable que notoriamente se independiza de la condición de consumidor, incluso de la de contratante (por ejemplo. el "okupa" del RD ley 37/2020). No tiene mucho sentido discutir, empero, si estos sujetos se encuentran incluidos en la síntesis conceptual del art. 3.2 TRLGDCU, porque la vulnerabilidad COVID es específica de cada norma, va acompañada de sus específicas consecuencias y no pretende aplicación generalizada fuera de su estricto marco normativo. 


\section{El consumidor vulnerable como destinatario de prácticas comerciales, publicidad e información precontractual}

Es fuera de las relaciones contractuales ( $y$, por tanto, fuera del art. 3.2 TRLGDCU) donde existen medidas adecuadas para proteger al consumidor vulnerable.

Ya se ha indicado como, para determinar si una práctica comercial es desleal, se evaluará desde la perspectiva del miembro medio de ese grupo vulnerable de destinatarios de la práctica (art. 4.3 LCD).

Igualmente, la consideración de consumidor vulnerable debe tomarse en consideración para determinar la información que ha de facilitárseles en el etiquetado y presentación de los bienes o servicios (nuevo art. 18.2 TRLGDCU), las comunicaciones comerciales y la información precontractual (nuevos arts. 19.6 y 20.2 TRLGDCU).

Una última reflexión, en relación con el control de transparencia de las cláusulas predispuestas que versan sobre el objeto principal del contrato. Conforme a una consolidada doctrina, para que la cláusula sea transparente el consumidor debe conocer las consecuencias económicas y jurídicas de la cláusula. Tradicionalmente el TJUE y el TS han entendido que el empresario debe facilitar esa información, pero la jurisprudencia más reciente del TS admite que el consumidor puede conocer esos datos por otras vías. La pregunta que cabe formular es la siguiente: ¿tiene el empresario que ajustar la información que facilita a las características de cada consumidor? ¿Ha de tener en cuenta, por ejemplo, que el consumidor es un analfabeto funcional o una persona anciana con dificultades cognitivas? La doctrina clásica del TS es que el consumidor debe conocer cierta información, lo que exige al empresario adaptar la información suministrada a cada concreto consumidor. Pero la doctrina más reciente del TS (sobre la cláusula IRPH y los acuerdos novatorios sobre cláusula suelo) sostiene que basta con que el empresario informe de cierta información (estandarizada) para que el consumidor medio (normalmente informado y razonablemente atento y perspicaz) pueda entenderla.

Tras la reforma del TRLGDCU, cabe preguntarse lo siguiente: para superar el control de transparencia material, ¿debe el empresario facilitar la información precontractual adaptada a las circunstancias particulares del destinatario (consumidor vulnerable) de forma que se asegure su adecuada comprensión, como requiere el nuevo art. 20.2 TRLGDCU?

\section{Bibliografía}

MARÍN LÓPEZ, M. J., "El «nuevo» concepto de consumidor y empresario tras la Ley 3/2014, de reforma del TRLGDCU", Revista CESCO de Derecho de Consumo, 2014, no 9 , pp. 9 y ss. 
MARÍN LÓPEZ, M. J., "¿Es consumidor el que adquiere un bien con ánimo de lucro? La doctrina del Tribunal Supremo contenida en la sentencia de 16 de enero de 2017", Revista CESCO de Derecho de Consumo, 2017, n 20, pp. 204 y ss.

MARÍN LÓPEZ, M. J., "Concepto de consumidor y préstamo para financiar una futura actividad empresarial", Cuadernos Civitas de Jurisprudencia Civil, Madrid. Ed. Civitas, 2020, no 112, § 2928, pp. 269 y ss. 